\title{
FOTOPROTETOR EM MUDAS DE CAFÉ ARÁBICA
}

\author{
Marcelo Marques Cobra', Kleso Silva Franco Junior ${ }^{1}$, Rafael Tadeu dos Santos ${ }^{2}$ \\ ${ }^{1}$ Centro Superior de Ensino e Pesquisa de Machado - MG (CESEP) Avenida Dr. Athaide Pereira de Souza, 730 - Centro - Machado - \\ MG, CEP $37.750-000$ \\ ${ }^{2}$ Grupo Grão de Ouro, Av. Alberto Vieira Romão, 2759 - Distrito Industrial - Alfenas - MG, CEP 37.135-516
}

*Autor para correspondência: Kleso Silva Franco Junior, kleso.junior@yahoo.com.br

\begin{abstract}
RESUMO: O café arábica é originário da Etiópia, cultivado na sua origem em sub bosques. Nos últimos anos estamos observando instabilidades climáticas nas principais regiões de cultivo do café no Brasil, assim sendo alguns danos tem sido observado na cultura, entre eles a escaldadura. Objetiva-se neste trabalho avaliar a aplicação de cálcio em mudas de café arábica e o seu efeito em minimizar os danos por escaldadura e temperatura na folha. $O$ experimento foi realizado na Fazenda Experimental da Grão de Ouro em Alfenas MG, com mudas de café arábica, cultivar Arara. Os tratamentos foram com uso de carbonato de cálcio $\left(\mathrm{CaCO}_{3}\right)$, Cal Virgem (CaO), ambos com uma dosagem de 2,4 kg.ha-1, com uma e 2 aplicações e o tratamento controle sem aplicação de cálcio. Os parâmetros avaliados foram a temperatura foliar, escaldadura e desenvolvimento de plantas. 0 delineamento experimental utilizado foi em blocos ao acaso com 4 repetições, totalizando 20 parcelas experimentais com 20 plantas por parcela. Os dados foram submetidos à análise de variância, por regressão e comparações de médias, teste de Tukey, a nível de 5\% de significância, por meio do software estatístico SISVAR. A aplicação de Carbonato de cálcio $\mathrm{CaCO}_{3}$ nas folhas, desempenhou uma ação de proteção, minimizando os efeitos das altas temperaturas, estresse solar e escaldaduras, permitindo um melhor desenvolvimento.
\end{abstract}

PALAVRAS CHAVES: Cálcio, Escaldadura, Temperatura.

\section{PHOTOPROTECTOR IN ARABICA COFFEE SEEDLINGS}

ABSTRACT: Arabica coffee is originally from Ethiopia, cultivated in its origin in understory. In recent years we have been observing climatic instability in the main coffee growing regions in Brazil, so some damage has been observed in the crop, including scalding. It is important to evaluate the application of calcium in Arabica coffee seedlings in this work and its effect in minimizing the damage caused by scalding and temperature in the leaf. The experiment was carried out at the Experimental Farm of Grão de Ouro in Alfenas MG, with Arabica coffee seedlings, cultivar Arara. The treatments were using calcium carbonate $\left(\mathrm{CaCO}_{3}\right)$, Virgin Lime $(\mathrm{CaO})$, both with a dosage of $2.4 \mathrm{~kg} \cdot \mathrm{ha}^{-1}$, with one and two applications and the control treatment without calcium application. The parameters evaluated were leaf temperature, scalding and plant development. The experimental design used was a randomized block with 4 replications, totaling 20 experimental plots with 20 plants per plot. The data submitted to analysis of variance, by regression and comparisons of means, Tukey's test, at the level of $5 \%$ of significance, using the statistical software SISVAR. The application of $\mathrm{CaCO}_{3}$ calcium carbonate on the leaves, performed a protective action, minimizing the effects of high temperatures, solar stress and scalding, allowing a better development.

KEYWORDS: Calcium, Scalding, Temperature.

\section{INTRODUÇÃO}

$O$ café arábica (Coffea arabica L.) é originário de áreas elevadas do sul da Etiópia, com temperatura ambiente entre 17 e $19^{\circ} \mathrm{C}$ e precipitação entre $1500 \mathrm{a}$ $1800 \mathrm{~mm}$ (Rocha et al., 2009)

As plântulas de café desenvolvem melhor à sombra que a pleno sol. A temperatura influência a formação do dossel vegetativo. A temperaturas ideal para cultura deve estar entre $24-28^{\circ} \mathrm{C}$, visto que em temperaturas baixas o cafeeiro não vegeta (Morais, 2011).

Por ser uma bebida muito consumida em cultura mundial, se torna fundamental, diminuir os efeitos negativos dos fatores temperatura, fotoperíodo, 
irradiância, irrigação e nutrientes, pois qualquer erro cometido no desenvolvimento da cultura poderá refletir negativamente durante a vida da cultura (Baliza et al.,2009).

Com as mudanças climáticas observadas neste último século, teme-se que a necessidade de adaptação ao ambiente seja muito intensa e com isso tenha agravada uma redução drástica de produção (Venturin et al., 2013). O tempo seco com pouca chuva e com altas temperaturas provoca 0 amarelecimento $\mathrm{e}$ a queima de folhas e frutos, com efeito na escaldadura do café (Matiello, 2015).

Muitos cientistas preveem que a temperatura média do ar no Planeta poderá aumentar entre $2^{\circ} \mathrm{C}$ e $4,5^{\circ} \mathrm{C}$, além de alterações na distribuição da precipitação (Poppe e Santos 2008).

A propagação do cafeeiro passa pela formação de mudas que devem ser de cultivares produtivas, bem adaptadas, sadias e vigorosas, plantadas em períodos adequados para o sucesso do empreendimento. A qualidade das mudas é influenciada diretamente pela formação da estrutura do sistema radicular e da parte aérea da planta, consequentemente, influi no comportamento da planta no campo. Quando esta etapa é bem conduzida, tem-se uma atividade mais sustentável, com maiores produtividades e com menores custos. (Baliza et al., 2010).

A produção de mudas de café segue uma tradição de formar mudas a $50 \%$ de sombreamento, porém com o passar dos anos alguns viveiristas e produtores vêm testando formar mudas á pleno sol, visando ter uma economia (Paiva, Guimarães e Souza, 2001).

Para proporcionar sustentabilidade a todo ciclo de produção de mudas é essencial que o manejo de condução das mudas no viveiro seja planejado e conduzido com intuito de aperfeiçoar a germinação e crescimento inicial das plântulas (Tomaz et al, 2012).

Segundo Tatagiba, Pezzopone e Reis (2010) a adaptação das plantas ao ambiente de luz depende do ajuste do seu aparelho fotossintético, de modo que a luminosidade ambiental seja utilizada da maneira mais eficiente possível, pois o crescimento pode estar relacionado a habilidade de adaptação das plântulas as condições de intensidade luminosa do ambiente.

Segundo Dias (2015), a extensa exposição das mudas à luz solar plena, em folhas o metabolismo fotossintético tem se inibido. Outros estresses, como altas temperaturas ou estresse hídrico, pode levar à fotoinibição crônica e à fotooxidação devido à maior geração de espécies reativas de oxigênio (EROs), que danificam o aparelho fotossintético. Estes efeitos são conhecidos como escaldadura ou queima de sol, que causa danos nas folhas e em outros órgãos fotossintetizantes, como frutos verdes.

Escaldadura é um dano fisiológico que ocorre sob duas formas: a clorose, provocada pela degradação da clorofila e a necrose, devido à morte dos tecidos (Matiello, 2015)

Nos últimos anos a cafeicultura tem sofrido com alguns fenômenos climáticos adversos, proporcionando alterações nas temperaturas, chuvas (irregularidade no ciclo, distribuição e volume), tal fato afeta o crescimento vegetativo e a produtividade sendo um dos efeitos mensuráveis a escaldadura (Rocha et al., 2009).

Nas condições de alta temperatura e incidência solar a planta absorve uma quantidade de energia do sol, que não consegue dissipá-la, provocando uma série de injúrias anatômicas nas folhas e frutos, como clorose seguida de necrose, prejudicando a produtividade (Matiello, 2015).

Segundo Dias et al. (2015) altos níveis de irradiância provocaram foto-oxidação e sintomas de escaldadura nas folhas dos cafeeiros, de forma mais intensa nas plantas deficientes em Mg e naquelas que recebem doses excessivas deste nutriente.

Desta forma uma muda bem formada leva consigo toda reserva de nutrientes e uma estrutura com resistência que suportar as adversidades na hora do plantio no campo (Paiva et al., 2001).

O carbonato de cálcio vem sendo usado para proteção dos frutos de maçãs contra queimaduras de sol, como é um fruto comercializado in natura a qualidade visual torna-se um atributo atrativo, mostrando-se uma opção para preservar a qualidade dos frutos (Martin et al., 2017).

Assim o objetivo do presente trabalho é avaliar a aplicação de cálcio em mudas de café arábica e 0 seu efeito em minimizar os danos por escaldadura e temperatura na folha.

\section{MATERIAL E MÉTODOS}

0 experimento foi conduzido no Centro Experimental da empresa Grão de Ouro Agronegócios 
Ltda, situado na Fazenda Primavera - latitude: $21^{\circ} 44^{\prime}$ 67, 92.06" S e longitude: $46^{\circ} 01^{\prime}$ '08.74" W, altitude 789 m, Rodovia Domingo Ribeiro Resende, BR491, KM 171, sentido Alfenas/Areado.

A temperatura média anual desta região é de $19,6^{\circ} \mathrm{C}$, precipitação média anual de $1.592,7 \mathrm{~mm}$ (BRASIL, 1992). O clima da região é classificado, segundo Köppen, como do tipo Cwa, mas apresenta características de Cwb, com duas estações distintas: inverno (abril a setembro) e verão (outubro a março). As mudas de café arábica (Coffee arabica L.) utilizadas foram da cultivar Arara, as quais foram transplantadas para os vasos com quatro pares de folhas. No período de adaptação das mudas (30 dias), o solo foi mantido com umidade próximo da capacidade de campo. Após as mudas adaptadas a irrigação foi cessada. Os tratamentos foram a aplicação de carbonato de cálcio $\left(\mathrm{CaCO}_{3}\right)$ contendo 25\% Ca, Cal Virgem (CaO) contendo 60\% $\mathrm{Ca}$, ambos com uma dosagem de $2,4 \mathrm{~kg} \mathrm{ha}^{-1}$. com uma e 2 aplicações e o tratamento controle sem aplicação de cálcio. As aplicações foram realizadas com pulverizador costal elétrico bateria Yamaho Ft16, com vazão de 4 litros por minuto e volume de calda de 80 litros por hectare, a primeira aplicação foi realizada 15 dias após o transplante e a segunda aplicação com intervalo de 60 dias
A temperatura foliar foi avaliada com um termômetro infravermelho com mira laser - modelo WT 550 , sempre as $11 \mathrm{~h}$, iniciando um dia após primeira a aplicação, efetuando esta mediação por 90 dias, e os dados sendo tabulados por média a cada 21 dias.

As avaliações de escaldadura e desenvolvimento de plantas foram iniciadas trinta dias após a primeira aplicação. $\mathrm{Na}$ avaliação do desenvolvimento das mudas, utilizou-se trena e paquímetro. Para avaliar a escaldadura e a mortalidade de plantas foi realizada a observação visual. 0 delineamento experimental utilizado foi em blocos ao acaso com 5 tratamentos, 4 repetições, totalizando 20 parcelas experimentais. Foram utilizadas 20 plantas por parcela, perfazendo um total de 400 plantas. Os dados coletados foram submetidos à análise de variância, por regressão e comparações de médias realizadas pelo teste de Tukey, a nível de $5 \%$ de significância, por meio do software estatístico SISVAR® (Ferreira, 2014).

\section{RESULTADOS E DISCUSSÃO}

Os dados obtidos pela avaliação do levantamento da temperatura com termômetro de infra vermelho nas folhas das mudas de café evidenciam que houve diferença estatística entre os tratamentos, conforme podemos ver na tabela 1.

Tabela 1. Resultados médios de Temperaturas $\left({ }^{\circ} \mathrm{C}\right)$, em folhas de mudas de café.

\begin{tabular}{lllllc}
\hline Tratamentos & $\mathbf{1}^{\mathbf{0}}$ Média & $\mathbf{2}^{\mathbf{0}}$ Média & $\mathbf{3}^{\mathbf{0}}$ Média & $\mathbf{4}^{\mathbf{0}}$ Média & Média geral \\
\hline $\mathrm{CaCO}_{3}$ - 1 Aplicação & $31,79 \mathrm{~A}$ & $30,98 \mathrm{~A}$ & $32,18 \mathrm{~A}$ & $32,87 \mathrm{~A}$ & $31,96 \mathrm{~A}$ \\
$\mathrm{CaO}-1$ Aplicação & $33,80 \mathrm{~B}$ & $33,70 \mathrm{~B}$ & $33,89 \mathrm{~B}$ & $35,00 \mathrm{~B}$ & $34,10 \mathrm{~B}$ \\
$\mathrm{CaCO}_{3}-2$ Aplicações & $31,66 \mathrm{~A}$ & $31,47 \mathrm{~B}$ & $31,58 \mathrm{~A}$ & $32,46 \mathrm{~A}$ & $31,79 \mathrm{~A}$ \\
$\mathrm{CaO}-2$ Aplicações & $33,90 \mathrm{~B}$ & $34,21 \mathrm{C}$ & $33,73 \mathrm{~B}$ & $34,89 \mathrm{~B}$ & $34,18 \mathrm{~B}$ \\
Testemunha & $34,51 \mathrm{~B}$ & $34,07 \mathrm{C}$ & $33,84 \mathrm{~B}$ & $34,96 \mathrm{~B}$ & $34,35 \mathrm{~B}$
\end{tabular}

Cv $(\%)=2,4$

Médias seguidas da mesma letra, na coluna, não diferem estatisticamente pelo teste de Tukey a $5 \%$

Em relação aos efeitos das temperaturas na fotoproteção, foi possível verificar que os resultados foram iguais estatisticamente para as temperaturas nos tratamentos com $\mathrm{CaCO}_{3}$ em 1 aplicação e 2 aplicações gerando os menores valores de temperatura, ambos respectivamente $31,96{ }^{\circ} \mathrm{C}$ e $31,79^{\circ} \mathrm{C}$. De acordo com ALFONSI, (2008) o excesso de luz acentua a bienalidade do cafeeiro além de causar a escaldadura em folhas do cafeeiro, danificando 0 aparelho fotossintético das mesmas.
Assim se mostrando eficientes na aplicação como fotoprotetores, pois foram capazes de absorver, refletir consequentemente proporcionar menor estresse a perda de água pelas folhas (MARTIN et. al, 2017). O recurso fotoprotetor permitem as folhas realizarem melhor a fotorrespiração, necessária em uma temperatura ideal. MORAIS, (2011) percebe-se que a temperatura ideal seria de $24-28^{\circ} \mathrm{C}$ para que esta realizasse seu processo de desenvolvimento vegetativo. 
De acordo com (ROCHA et al.,2009) a radiação quando chega até a planta, de forma refletida, parte é absorvida e parte é redistribuída, portanto, os tratamentos da segunda avaliação $\mathrm{CaO}$ e Testemunha passou por uma fotoinibição, que intensificou a alta temperatura levando a planta ao estresse, e causando a foto-oxidação no aparelho fotossintético, sendo manifestados em folhas e em frutos conhecidos como escaldadura ou queima do sol (DIAS,2015).
Os dados refletiram na incidência de escaldadura, onde foi observado as diferenças significativas na aplicação de cal virgem $\mathrm{CaO}$, em relação a incidência de escaldadura nas folhas em comparação ao tratamento controle.

Em relação a incidência de escaldadura, os resultados apresentados na Tabela 2 de acordo com análise de variância, mostra que houve diferenças estáticas.

Tabela 2. Resultados médios de escaldadura em porcentagem (\%), em folhas de mudas de café.

\begin{tabular}{llc}
\hline Tratamentos & & Escaldadura (lesão) \\
\hline $\mathrm{CaCO}_{3}$ & 1aplicação & $25,00 \mathrm{~B}$ \\
$\mathrm{CaO}$ & 1 aplicação & $34,00 \mathrm{~B}$ \\
$\mathrm{CaCO}$ & 2 aplicações & $15,00 \mathrm{~A}$ \\
$\mathrm{CaO}$ & 2 aplicações & $37,50 \mathrm{~B}$ \\
Testemunha & & $55,00 \mathrm{C}$ \\
\hline
\end{tabular}

$\mathrm{CV}(\%)=47,94$

${ }^{*}$ Médias seguidas da mesma letra, na coluna, não diferem estatisticamente pelo teste de Tukey a 5 \%

$\mathrm{O}$ tratamento que apresentou o menor número de lesões de escaldadura nas folhas, foi $0 \mathrm{CaCO}_{3}$ com 2 aplicações, seguido de todos os tratamentos à base de $\mathrm{Ca}$ que tiveram eficiência intermediária para a escaldadura e a testemunha com um número elevado de lesões.

Segundo Dias et al. (2015), os níveis de irradiância $\left(80\right.$ e $320 \mu \mathrm{mol}$ fóton $\mathrm{m}^{-2} \mathrm{~s}^{-1}$ ) provocam foto oxidação e sintomas de escaldadura em folhas de cafeeiros (DIAS et al., 2015). 0 mesmo pode ser observado com a morte dos tecidos sob a forma de clorose e necrose (MATIELLO, 2015).

De acordo (Martin et al., 2017) Os produtos à base de carbonato de cálcio formam películas de finas partículas minerais que atuam como barreiras. Protegendo os frutos contra queimadura do sol, diminuindo assim o estresse solar e consequentemente reduzindo a ocorrência do distúrbio.

A aplicação de carbonato de cálcio $\mathrm{CaCO}_{3}$ nas folhas, desempenhou uma ação de proteção, minimizando os efeitos das altas temperaturas, estresse solar e escaldaduras, permitindo a não formação de escaldadura e manchas necróticas.

\section{AGRADECIMENTOS}

Agradecemos ao Grupo Grão de Ouro e ao CESEP Centro Superior de Ensino e Pesquisa de
Machado MG, pelo apoio dispensado na realização deste trabalho.

\section{REFERÊNCIAS BIBLIOGRÁFICAS}

Alfonsi, E.L. Uso de Índices Fenológicos em Modelos de Previsão de Produtividade do café. Tese (Doutorado), Universidade De São Paulo Escola Superior de Agricultura "Luiz de Queiroz". Área de Concentração Fitotecnia, Piracicaba, p. 1-105, 2008. Disponível em <http://www. teses.usp.br/teses/disponiveis/11/11136/tde-22072008155558/pt-br.php>. Acesso em: 05 jun. 2018

Baliza, D.P. Antecipação da Produção do cafeeiro (Coffea arabica L.) com a utilização de diferentes tipos de mudas. O Repositório Institucional da Universidade Federal de Lavras (RIUFLA), Lavras, 2009, 1-44.

Disponível em: <http://repositorio.ufla.br/ bitstream/1/4170/1/disserta $\% c 3 \% 87 \% \quad$ c3\%830 antecipa \% c 3\% a $7 \%$ c $3 \%$ a $30 \% 20$ da $\% 20$ produ \%c3\%a $7 \%$ c3 $\%$ a 30\% 20 do $\% 20$ cafeeiro 20 coffea $\% \quad 20$ arabica $\% \quad 201 . \% \quad 20$ com $\%$ 20utiliza $\%$ c3\% a7\% c3\% a30\% 20de\% 20diferentes $\%$ 20tipos $\%$ 20de $\%$ 20mudas.pdf>. Acesso em: 15 mai. 2018

Baliza, D.P.; Ávila, F.W.; Carvalho, J.G.; Guimarães, R.J.; Passos, A.M.A.; Pereira, V.A. Crescimento e 
nutrição de mudas de café controlado pela substituição do potássio pelo sódio. Revista Coffee Science, 2010 $5,3,272-282$.

Disponível em: <https://info.cnptia.embrapa.br/digital/ bitstream/item/42551/1/na-k-cafeeiro-alexandre.pdf>. Acesso em: 29 abr. 2018

Dias, K. G. L.; Guimaraes, P. T. G; Furtini Neto, A.E; Silveira, H.R.O.; Lacerda, J.J.J. Effect of Magnesium on Gas Exchange and Photosynthetic Efficiency of Coffee Plants Grown under Different Light Levels, Agriculture, 2017, 7, 10, 2-11.

Disponível em:< https://www.mdpi.com/20770472/7/10/85 >. Acesso em: 20 fev. 2020.

Dias, K.G.L.; Guimarães, P.T.G.; Furtini Neto, A.E.; Oliveira, C.H.C.; Tiburcio, G.S.; Dias, C.M.L. Dinâmica de carboidratos e escaldadura em cafeeiros supridos com MG. XXXI Congresso Brasileiro de Ciência do Solo Centros de Convenções, Natal/RN, p. 1-3, ago. 2015. Disponivel em:<https://www.sbcs.org.br/cbcs2015/ arearestrita/arquivos/ 392.pdf $>$. Acesso em: 27 mai. 2018.

Ferreira, D.F. Sisvar: um guia dos seus procedimentos de comparações múltiplas Bootstrap. A Ciência e agrotecnologia, 2014, 38, 2, 109-112.

Martin, M.S.; Petri, J.L.; Sezerino, A.A.; Gabardo, G.C.; Fenili, C.L. Incidência de queimadura de sólidos em macieiras submetidas a aplicações de carbonato de cálcio. Revista da $14^{a}$ Jornada da Pós graduação e Pesquisa- Congrega, Santa Catarina, p. 1-11, set. 2017.

Disponível em: <http://trabalhos.congrega.urcamp.edu. br/index.php/14jpgp/article/view/1902>. Acesso em: 12 jun. 2018

Matiello, J.B. Escaldadura em folhas e frutos do cafeeiro. Café Point, 2015.

Disponivel em: <https://www.cafepoint.com.br/noticias/ tecnicas-de-producao/escaldadura-em-folhas-e-frutosdo-cafeeiro-93382n.aspx>. Acesso em: 24 jun. 2018

Matiello, J.B.; Santinato, R..; Garcia, A.W.R.; Almeida, S.R.; Fernandes, D.R. Cultura de café no Brasil: Novo
Manual de Recomendações. 2002. Rio de Janeiro, $1-387$.

Morais, F. C. Morfologia, fisiologia e fenologia do cafeeiro. Scribd, Uberlândia, 2011, 1-23.

Disponível em: <https://pt.scribd. com/doc/187133401/ morfologia-fisiologia-e-fenologia-do-cafeeiro>. Acesso em: 30 mai. 2018.

Paiva, L. C; Guimarães, R. J; Souza, C.S. Aspecto fisiológico de mudas de cafeeiro (Coffea arabica L.) formadas a pleno sol. In: II Simpósio de Pesquisa dos Cafés do Brasil, 2001, 1-10.

Disponível em: <http://www.sbicafe.fv.br/bitstream/ handle/123456789/1238/155585_art002f. pdf?sequence $=1$ \&isall owed=y>. Acesso em: 28 mai. 2018

Paiva, L.C.; Guimarães, R.J.; Castro, E.M.; Souza, C.S. Aspectos da anatomia foliar de mudas de cafeeiro (Coffea arabica L.) submetidas a diferentes níveis de sombreamento. In: Simpósio de Pesquisa dos Cafés do Brasil, Vitória, ES, 2001, 1619-1625.

Disponivel em: <http://www.sbicafe.ufv.br/bitstream/ handle/123456789/1295 /155585_art224f. pdf?sequence=1\&isallowed=y>. Acesso em: 12 jun. 2018

Poppe, M.K.; SANTOS, M.M. Mudança climática rumo a um novo acordo mundial. In: III Conferência Regional Sobre Mudança Global: América Do Sul, São Paulo, 2008, 1-240. Disponível em: <http://www.iea.usp.br/publicacoes/textos/ relatorio3confregmudancasglobaisal. pdf>. Acesso em: 24 jun. 2018

Rocha, V.P.C.; Ribeiro Filho, C.; Shigueoka, L.H.; Alegre, C.R.; Colombo, L.A.; Del Grossi, L.; Azevedo, J.A.; Sera, T.; Bosquesi, E.P. Avaliação da escaldadura de sol em cultivares de café plantado em diferentes espaçamentos. In: VI Simpósio de Pesquisa dos Cafés do Brasil, Araxá. 2009.

Disponível em: <http://www.sbicafe.ufv.br/ bitstream/handle/123456789/2656/401. pdf?sequence=1\&isallowed=y>. Acesso em: 20 jun. 2018 
Tatagiba, S.D.; Pezzopane, J.E.M.; Reis, E.F. Disponível em: <https://www.researchgate.net/ Crescimento Vegetal de Café Arábica (Coffea arabica publication $\quad$ /306396266_producao_de_mudas L.) Submetidas a Diferentes Níveis de Sombreamento. de_qualidade_base_para_a_sustentabilidade_da_ Revista Coffee Science, 2010, 5, 3, 251-261. lavoura_cafeeira>. Acesso em: 30 abr. 2018

Disponível em: <file: ///c:/users/ cliente/downloads/1311274-1-pb.pdf>. Acesso em: 30 mai. 2018

VENTURIN, R.P.; Silva, V.A.; Cunha, R.L.; Volpato, M.M.L.; Chalfoun, S.M.; Carvalho, G.R.; Carvalho, V.L. A Pesquisa e como Mudanças Climáticas

Tomaz, M.A.; Amaral, J.F.T.; Jesus Junior, W.C.; Fonseca, A.F.A.; Ferrão, R.G; Martins, L.D.; Rodrigues, nas Cafeicultura. Informe Agropecuário (Semana Internacional do Café), 2013, 34, 34-43.

W.N. Produção de mudas de qualidade: base para a sustentabilidade da lavoura cafeeira. Inovação, difusão e integração: base para sustentabilidade da Disponível em:<http://www.epamig.ufla.br /geosolos/ cafeicultura, Alegre, Es, 71-87, 2012. 